\title{
Optimal diuretic strategies in heart failure
}

\author{
Sarabjeet S. Suri, Salpy V. Pamboukian \\ Division of Cardiovascular Diseases, University of Alabama at Birmingham, Birmingham, AL, USA \\ Contributions: (I) Conception and design: SS Suri; (II) Administrative support: SV Pamboukian; (III) Provision of study materials or patients: Both \\ authors; (IV) Collection and assembly of data: Both authors; (V) Data analysis and interpretation: Both authors; (VI) Manuscript writing: Both \\ authors; (VII) Final approval of manuscript: Both authors. \\ Correspondence to: Dr. Sarabjeet S. Suri, MD. University of Alabama at Birmingham, 1900 University Blvd, THT 321, Birmingham, AL 35223, USA. \\ Email: sssuri@uabmc.edu.
}

\begin{abstract}
Heart failure (HF) is one of the major causes of morbidity and mortality in the world. According to a 2019 American Heart Association report, about 6.2 million American adults had HF between 2013 and 2016, being responsible for almost 1 million admissions. As the population ages, the prevalence of HF is anticipated to increase, with 8 million Americans projected to have HF by 2030, posing a significant public health and financial burden. Acute decompensated HF (ADHF) is a syndrome characterized by volume overload and inadequate cardiac output associated with symptoms including some combination of exertional shortness of breath, orthopnea, paroxysmal nocturnal dyspnea (PND), fatigue, tissue congestion (e.g., peripheral edema) and decreased mentation. The pathology is characterized by hemodynamic abnormalities that result in autonomic imbalance with an increase in sympathetic activity, withdrawal of vagal activity and neurohormonal activation (NA) resulting in increased plasma volume in the setting of decreased sodium excretion, increased water retention and in turn an elevation of filling pressures. These neurohormonal changes are adaptive mechanisms which in the short term are associated with increased contractility of the left ventricular (LV) and improvement in cardiac output. But chronically, the failing heart is unable to overcome the excessive pressure and volume leading to worsening HF. The primary symptomatic management of $\mathrm{ADHF}$ includes intravenous (IV) diuresis to help with decongestion and return to euvolemic status. Even though diuretics have not been shown to provide any mortality benefit, they have been clinically proven to be of significant benefit in the acute decompensated phase, as well as in chronic management of HF. Loop diuretics remain the mainstay of therapy for symptomatic management of HF with use of thiazide diuretics for synergistic effect in the setting of diuretic resistance. Poor diuretic efficacy has been linked with higher mortality and increased rehospitalizations.
\end{abstract}

Keywords: Heart failure (HF); diuresis; diuretic resistance

Submitted Jun 10, 2020. Accepted for publication Aug 07, 2020.

doi: 10.21037/atm-20-4600

View this article at: http://dx.doi.org/10.21037/atm-20-4600

\section{Renal physiology}

Diuretics act on kidneys, and as such, understanding renal physiology is pivotal to understanding the role of diuretic therapy in treating heart failure (HF). Kidneys receive about $20 \%$ of cardiac output which corresponds to about $1 \mathrm{~L} /$ minute. The glomerular filtration rate (GFR) is the amount of fluid filtered from glomeruli into Bowman's capsule per unit time expressed as $\mathrm{mL} / \mathrm{min} / 1.73 \mathrm{~m}^{2}$. Filtration fraction $(\mathrm{FF})$ is the amount of renal plasma flow (RPF) that is filtered across glomerulus (GFR/RPF). It averages about 20\%. Extent of chronic kidney disease (CKD) is based on the GFR: stage 1 GFR $>90$, stage 2 GFR $60-89$, stage 3a 45-59, stage 3b $30-44$, stage $415-29$, and stage $5<15$. CKD has been demonstrated to be an independent risk factor for poor outcomes, morbidity, and mortality in HF (1).

Kidney autoregulation works to maintain a normal GFR. Renin-angiotensin-aldosterone system (RAAS) 
plays an important role through a sequential downstream pathway leading to the activation of angiotensin II which is responsible for inducing vasoconstriction resulting in increased blood pressure, constricting efferent arterioles to increase FF in the setting of low renal blood flow (RBF), as well as the releasing aldosterone from the zona glomerulosa of the adrenal gland. It is also responsible for antidiuretic hormone $(\mathrm{ADH})$ secretion in the posterior pituitary gland which stimulates the insertion of the aquaporin channel in the collecting duct, responsible for water reabsorption (2).

The syndrome of HF decreases RBF as a consequence of increased venous pressure which leads to increased FF resulting in increased protein concentration in the peritubular capillaries, inadvertently leading to more sodium $\left(\mathrm{Na}^{+}\right)$and water reabsorption in the nephron proximally. Neurohormonal activation in HF, i.e., RAAS and sympathetic nervous system activation, occurs as a compensatory mechanism to maintain hemodynamics via salt and water retention and peripheral vasoconstriction. When these changes in persist in the chronic setting deleterious effects are induced, leading to further progression of $\mathrm{HF}(3)$.

\section{Defining acute decompensated HF (ADHF)}

Patients with AHDF can present with a myriad of presentations from volume overload to cardiogenic shocks requiring inotropic or mechanical circulatory support for rescue. Factors leading to exacerbation usually include dietary indiscretion, medication non-adherence, substance abuse, new cardiac disease (e.g., ischemia), arrhythmias, and progression of underlying cardiomyopathy. In many cases, the underlying cause of the exacerbation is unable to be identified. ADHF further contributes to the disease progression through left ventricle remodeling, multi organ failure including renal as well as liver dysfunction, pulmonary hypertension and right ventricular dysfunction.

Currently, we are most reliant on our physical exam, laboratory data and imaging to confirm an ADHF episode. Physical exam findings including S3 gallop, elevated jugular venous pressure (JVP), lung crackles, ascites, and bilateral pitting edema are seen in HF. Presence of an S3 gallop has a high specificity for ADHF. Elevated JVP above $8 \mathrm{~cm}$ has a $48 \%$ sensitivity and $78 \%$ specificity to detect congestion. Elevated JVP also correlates well with elevated left-sided filling pressures. Bilateral leg edema has $94 \%$ sensitivity but only $10 \%$ specificity for detecting congestion.

Combining the physical exam with laboratory data including biomarkers like elevated brain natriuretic peptide (BNP) helps further corroborate an ADHF episode. Normal natriuretic peptide levels $(B N P<100 \mathrm{pg} / \mathrm{mL}$, $\mathrm{N}$-terminal pro-BNP $<300 \mathrm{pg} / \mathrm{mL}$ ) are helpful in excluding $\mathrm{HF}$ as they carry a high negative predictive value for $\mathrm{ADHF}$ $(4,5)$. Imaging evidence of congestion, such as distended inferior vena cava and B lines in more than 3 lung fields on bedside ultrasound, can aid in establishing the diagnosis. A transthoracic echocardiogram to assess left ventricular (LV) dysfunction, LV dimension, ejection fraction (6). Right heart catheterization (RHC) remains the gold standard for diagnosis ADHF and to risk stratify the severity of the disease, but given its invasive nature and need for a trained physician to perform the procedure, it is difficult to perform on every patient. Furthermore, the Evaluation Study of Congestive Heart Failure and Pulmonary Artery Catheterization Effectiveness (ESCAPE) trial which evaluated the role of RHC in ADHF showed that overall mortality and hospitalization rates were not improved with the addition of routine RHC, when compared with physical exam alone (7).

Another dilemma while dealing with ADHF is establishing what euvolemia is. The term first defined in 1979, refers to the dry weight or ideal volume status to which diuresis is targeted. Targeting a "dry weight" has been one of the cornerstone strategies that is employed for measuring congestion relief in ADHF patients, but it actually has a poor correlation with decongestion. Post hoc analysis of Diuretic Optimization Strategy Evaluation Heart Failure (DOSEHF) and Cardiorenal Rescue Study in Acute Decompensated Heart Failure (CARESS-HF) trials has shown that despite aggressive diuresis, $35-40 \%$ of patients were still moderately congested at discharge (8). Hemoconcentration demonstrated by elevated hemoglobin/hematocrit or albumin levels during the latter part of the hospitalization has also been studied as another marker of decongestion (9).

Salt and fluid restriction has been seen as a nonpharmacological way to prevent ADHF. $\mathrm{Na}^{+}$restriction of less than $2 \mathrm{~g} /$ day and fluid restriction $<2 \mathrm{~L} /$ day are recommended to all patients with congestive $\mathrm{HF}$ and are endorsed by American Heart Association (AHA), Heart Failure Society of America as well as European Society of Cardiology. These guidelines are based on expert opinion, level of evidence C (10).

\section{Diuretics}

Any substance that increases micturition and water excretion 
Table 1 Loop diuretic dosing comparison $(11,13)$

\begin{tabular}{|c|c|c|c|c|}
\hline Characteristics & Furosemide (Lasix) & Torsemide & Bumetanide & Ethacrynic acid \\
\hline Bioavailability (\%) & $10-100$ & $80-100$ & $80-100$ & 100 \\
\hline Initial oral dosing (mg) & $20.0-40.0$ & $10.0-20.0$ & $0.5-1.0$ & 50.0 \\
\hline Relative potency & 40 & $10-20$ & 1 & NA \\
\hline Maximum dosing in 24 hours (mg) & 600 & 200 & 10 & 200 \\
\hline Duration of effect (hours) & $\sim 6$ & $6-16$ & $4-6$ & $\sim 6-8$ \\
\hline
\end{tabular}

IV, intravenous.

is referred to as a diuretic. Diuretics act by inhibiting $\mathrm{Na}^{+}$ reabsorption causing a decreased osmotic gradient across the tubule which limits water re-absorption, thereby promoting diuresis. Diuretics act on various transporters in the nephron, so they have to be secreted in the tubular lumen through the organic acid secretory pathway in order to "get to" the transporters.

\section{Loop diuretics}

Loop diuretics remain the mainstay of therapy for symptomatic management of HF. These are highly proteinbound organic anions, which are secreted across the proximal convoluted tubule where they act on sodiumpotassium chloride $(\mathrm{Na}-\mathrm{K}-2 \mathrm{Cl})$ channel in the thick ascending loop of Henle to inhibit $20-30 \%$ of the $\mathrm{Na}^{+}$ reabsorption that occurs here, thereby promote the delivery of $\mathrm{Na}^{+}$to distal tubule (11). Loop diuretics have a steep dose-response curve and threshold dose below which they do not produce any natriuresis. The threshold dose may be different for every patient, depending mostly on their GFR. A dose of $10 \mathrm{mg}$ may be enough to produce an adequate diuretic effect in someone with normal kidney function whilst a dose as high as 80 to $120 \mathrm{mg}$ might be needed to achieve a similar response in advanced kidney disease. They are also referred to as "high ceiling" diuretics because the progressive increase in their dose promotes more natriuresis but after a certain maximal dose the effect hits a plateau which is referred to as the "ceiling". Loop diuretics use has been shown to activate both the RAAS and sympathetic nervous system directly due to a decrease in intravascular volume and indirectly through inhibition of $\mathrm{NaCl}$ uptake in macula densa resulting in renin release, which may contribute to further progression of HF. The neurohormonal activation is dose independent (low vs. high dose of diuretics) as was seen in post hoc analysis (12).

Loop diuretics that are currently approved in the USA include furosemide, bumetanide, torsemide and ethacrynic acid (Table 1). These are sulfonamide drugs except for ethacrynic acid which is a non-sulfonamide diuretic. It is rarely used due to its significant ototoxicity and is reserved for patients with hypersensitivity or who have experienced adverse reactions to other medications.

Furosemide bioavailability differs and varies between $10-100 \%$ based on the formulations being used [oral $v s$. intravenous (IV)]. IV doses of furosemide are twice as potent as an oral formulation. It is the most common loop diuretic used but there is no clinical evidence of its superiority over other loop diuretics available. In contrast, torsemide and bumetanide have a higher bioavailability ranging from $80-100 \%$. Torsemide also has a longer halflife (6 hours) as compared to bumetanide or furosemide which is about $2-3$ hours.

TOrasemide In Congestive Heart Failure (TORIC) study compared the efficacy, tolerability and safety of Torsemide in comparison to furosemide. This was a nonrandomized, post-market surveillance study which found that not only torsemide was safe and well tolerated but it was also associated with $51.5 \%$ relative risk reduction in mortality, $59.5 \%$ reduction in cardiac mortality compared to furosemide (14). Several post-marketing studies have shown decreased hospitalization with torsemide use when compared to other diuretics. Considering which the overall cost of patients with ADHF who were treated with torsemide was significantly lower when compared with other diuretics. Even though the initial acquisition cost is higher for torsemide when compared to furosemide, the overall cost burden is lower (15). 
Since the half-life of loop diuretics is short, they often require frequent dosing (at least twice a day) to prevent post diuresis $\mathrm{Na}^{+}$reabsorption. Side effects of loop diuretics include ototoxicity, hypersensitivity reaction, metabolic alkalosis/contraction alkalosis, hyperuricemia, electrolyte abnormalities including hypokalemia, hyponatremia.

In the setting of ADHF, gastrointestinal absorption of oral diuretics is decreased due to gut edema and decreased duodenal blood flow contributing to resistance to oral therapy. IV administration bypasses gut edema and is more efficacious in settings where there is decreased absorption of oral medications $(13,16)$.

\section{Thiazide diuretics}

Thiazide diuretics act on the distal convoluted tubule, where about 3-5\% of $\mathrm{Na}^{+}$reabsorption occurs, by blocking $\mathrm{NaCl}$ cotransporter resulting in decreased $\mathrm{Na}^{+}$ and $\mathrm{Cl}^{-}$reabsorption and promoting the delivery of $\mathrm{Na}^{+}$ to collecting ducts. They impair kidneys diluting capacity and promote calcium reabsorption. They are often used in addition to loop diuretics to augment diuresis and help alleviate diuretic resistance. Commonly used thiazide diuretics include metolazone, hydrochlorothiazide, and chlorothiazide. Addition of thiazide type diuretic, chlorothiazide or metolazone as an add on therapy to loop diuretic was proven to be significant to augment diuresis in the setting of diuretic resistance and improved weight loss. No statistically significant difference was observed between oral metolazone $v s$. IV chlorothiazide $(17,18)$.

Combination diuretic therapy is associated various adverse effects including electrolyte abnormalities like hypokalemia, hyponatremia, hypomagnesemia, hypochloremia along with metabolic acidosis. They may also worsen renal dysfunction, cause hypotension and induce cardiac arrhythmias.

\section{Potassium-sparing diuretics}

Potassium-sparing diuretics work on the collecting duct in the nephron. These include eplerenone, amiloride, spironolactone, and triamterene. They are also referred to as "weak diuretics" as their site of action is collecting duct where only $3 \%$ of $\mathrm{Na}^{+}$reabsorption occurs. The collecting duct has epithelial $\mathrm{Na}^{+}$channels which are directly inhibited by amiloride and triamterene thus limiting $\mathrm{Na}^{+}$ reabsorption. Meanwhile, aldosterone receptor blockers (spironolactone and eplerenone) downregulate the $\mathrm{Na}^{+} /$
$\mathrm{K}^{+}$pump and epithelial $\mathrm{Na}^{+}$channels in the collecting duct which leads to decreased $\mathrm{Na}^{+}$reabsorption and secretion of $\mathrm{H}^{+} \& \mathrm{~K}^{+}$cations. These are not used as monotherapy for diuresis but used in combination with loop diuretics to augment their effect, as well as for maintenance of $\mathrm{K}^{+}$levels in patients. In contrast to other diuretics, spironolactone and eplerenone have been shown to have mortality benefit and reduce hospitalization in patients with systolic HF $(10,19)$. Additional beneficial effects of these drugs include reducing sympathetic activation and myocardial fibrosis.

Vasopressin receptor antagonist (e.g., tolvaptan) works by inhibiting V2 receptors in the collecting duct, blocking aquaporin channel insertion and increasing water excretion. Despite trends showing reduced dyspnea compared to placebo and increased weight loss tolvaptan has not been shown to increase the number of clinical responders at 24 hours when added to furosemide and therefore is not routinely used to treat $\operatorname{ADHF}(20,21)$.

Acetazolamide is a sulfonamide-based drug which works by inhibition of carbonic anhydrase enzyme in the proximal convoluted tubule leading to natriuresis as well as bicarbonate excretion. It is often used to correct contraction alkalosis caused by "over-diuresis".

Metabolic alkalosis is a common acid base disturbance associated with ADHF. It may occur due to volume overload, electrolyte disturbances, RAAS activation or as a consequence of diuresis. Acetazolamide may assist in reversing the alkalosis. But underlying reversible causes of alkalosis including electrolyte abnormalities, volume overload should be addressed first (22). The ongoing trial Acetazolamide in Decompensated heart failure with Volume OveRload (ADVOR) will investigate effects of acetazolamide combined with loop diuretic therapy on improving diuresis and congestion in $\operatorname{ADHF}(23)$.

\section{Dosing diuretic during acute decompensation}

There is a lack of substantial evidence on how to dose diuretics in ADHF. This is evident from the American College of Cardiology (ACC)/AHA guidelines, as they have a class I recommendation with level C of evidence (24). The DOSE trial involved 2 by 2 factorial design where 308 patients were randomly assigned to receive either a low dose or high dose of furosemide intravenously either via boluses which were administered twice day or via continuous infusion. Low doses were defined as the equivalent of the patient's home oral dose vs. high dose was 2.5 times of the home dose. High-dose diuretics were associated with better 
symptom relief than low-dose diuretics albeit at the cost of some renal dysfunction, meanwhile there was no significant difference in continuous versus bolus infusion (25). Even though some studies have found continuous infusion is better than bolus dosing but this was not seen in DOSE trial (26).

With ADHF, we recommend administering diuretics intravenously. Initial dosing will depend if the patients are diuretic naive or on chronic diuretic therapy. If naive, consider starting at low dose, e.g., furosemide 20 to $40 \mathrm{mg}$ twice daily. Meanwhile for patients on chronic therapy consider doubling the outpatient dose as the initial IV dose, administered twice daily. Further dosing should be titrated depending upon the patient's diuretic response as measured by urine output and reduction in signs and symptoms of congestion such has decreased dyspnea, reducing edema and lessening JVP. During diuresis, renal function and electrolytes should be frequently monitored, at least daily while hospitalized. Electrolyte derangements should be promptly corrected. Once effectively diuresed, patients should be transitioned to the lowest effective dose of oral diuretics to maintain euvolemia with the aim to add and titrate up guideline-directed medical therapy (Figure 1).

\section{Diuretic resistance}

Diuretic efficacy is defined by metrics of the patient's response post diuretic administration. It can be measured by urinary output or weight change post diuresis. Optimal diuretic response has been linked to prolonged eventfree survival. It is defined as at least $1.5-2 \mathrm{~L}$ of negative fluid balance or 1-2 pounds of weight decline over 24 hours post diuretic administration. Based on the definition referred to as above, diuretic resistance may be defined as an inadequate response and ineffective decongestion despite optimal diuretic therapy. There can be a myriad of reasons for diuretic resistance, management of which includes elucidating the underlying causes \& working to reverse them.

Oral loop diuretics such as furosemide do not work well when patients are in acute exacerbation due to decreased bioavailability and or absorption as a result gut edema, leading to poor absorption thus necessitating parenteral therapy for the resistance breakthrough $(13,16)$.

Loop diuretics are highly bound to plasma protein and are secreted across the proximal convoluted tubule by organic anion transporters. In patients with advanced kidney disease, uremic anions compete with loop diuretics for secretion across the proximal convoluted tubule. Metabolic acidosis inhibits this phenomenon as well. Thus, in patients with advanced kidney disease, higher doses are required to get to the threshold dose of loop diuretics.

Hypoalbuminemia also leads to increase drug distribution volume thereby limiting delivery to the kidneys. A metanalysis examined the role of administration of albumin along with furosemide revealed an increase in urine volume as well as $\mathrm{Na}^{+}$ excreted at 8 hours when co-administered but these changes were not statistically significant at 24 hours (27).

Intraabdominal hypertension due to excessive fluid overload and ascites may result in poor renal perfusion. In such cases, diuresis alone would be ineffective \& may lead to worsening of renal function. Paracentesis or ultrafiltration (UF) may be necessary to alleviate ascites without overly compromising renal function.

Post diuretic $\mathrm{Na}^{+}$retention has also been attributed as one of the causes of diuretic resistance, which corresponds to reabsorption of $\mathrm{Na}^{+}$as an adaptive measure by autoregulation in the kidneys to compensate for prior $\mathrm{Na}^{+}$ loss. Loop diuretics trigger the RAAS through multiple pathways. In a volume independent pathway, they cause the release of renin from afferent arteriole by inhibiting the $\mathrm{Na}$ $\mathrm{K}-2 \mathrm{Cl}$ channel in macula densa. With volume contraction, the RAAS is triggered. This eventually leads to more $\mathrm{Na}^{+}$ reabsorption as well as its avidity, leading to post diuretic $\mathrm{Na}^{+}$retention. Using a combination of two different class of diuretics can help overcome this.

Braking phenomenon refers to a diminished response to a dose of loop diuretic which initially produced an optimal response, usually due to adaption in the nephrons. Rat studies have shown that chronic administration of loop diuretic leads to hypertrophy and hyperplasia in epithelial cells of distal convoluted tubule, leading to more $\mathrm{Na}$ reabsorption and thereby causing a dullened response and a shift of the dose-response curve further down and right.

Another consideration for lack of diuretic response is that the patient does not truly have expanded intravascular volume due to ADHF. Edema may be due to third spacing, venous insufficiency or lymphatic edema which will not respond to diuretic therapy, making it appear that the patient has diuretic resistance. In these situations, escalating diuretic doses in an apparent diuretic resistant patient in an attempt to resolve edema may lead to significant renal impairment. In these cases, obtaining a hemodynamic evaluation using RHC may be very helpful in differentiation cardiac versus non cardiac edema.

Medications like non-steroidal anti-inflammatory drugs 


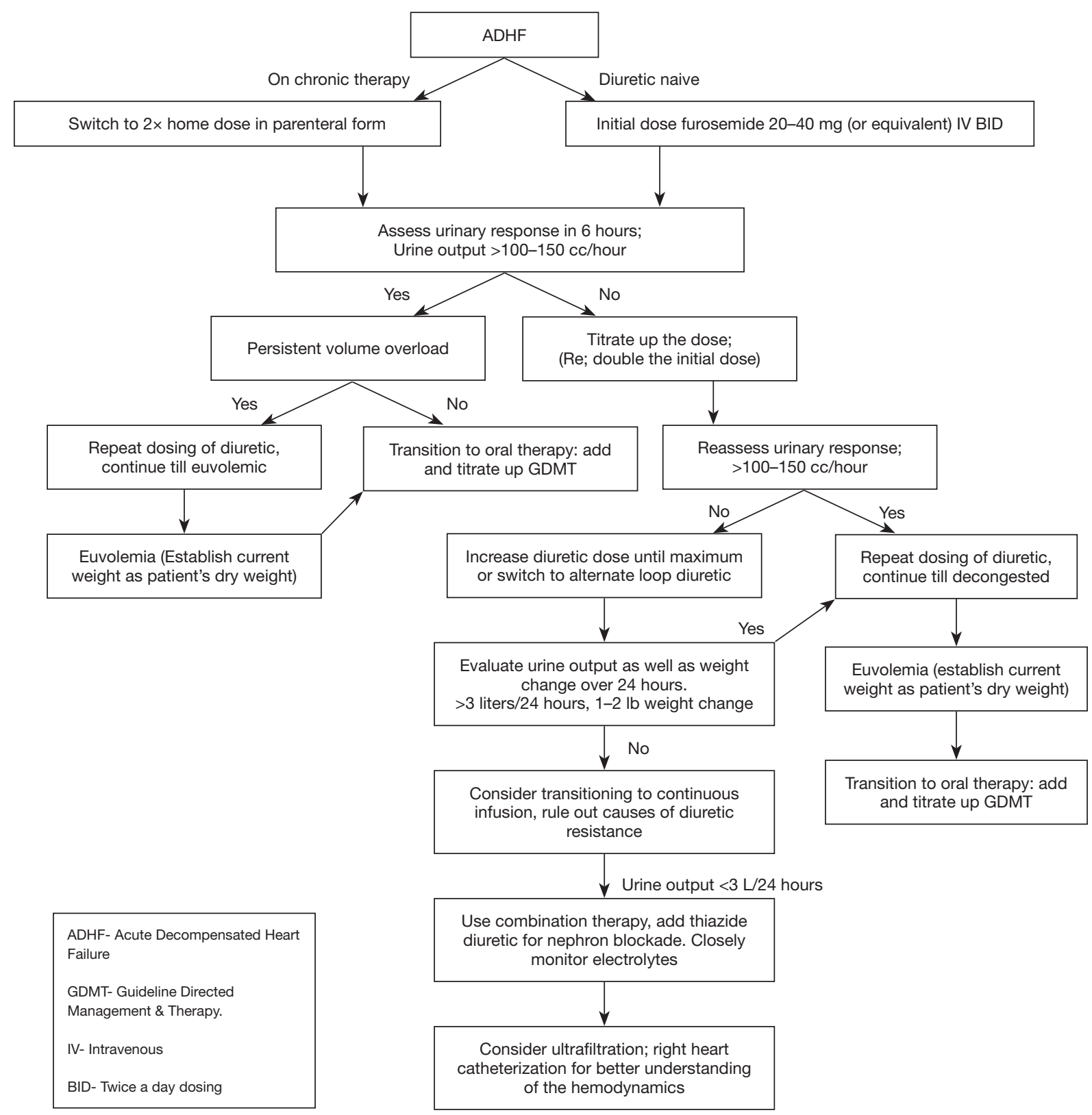

Figure 1 Schematic management of ADHF. ADHF, acute decompensated heart failure; GDMT, guideline directed management \& therapy; $\mathrm{IV}$, intravenous; BID, twice a day dosing.

(NSAIDs) can cause decrease prostaglandin synthesis thereby inhibiting renal vasodilation and lead to worsening hypertension as well as worsening of renal dysfunction. NSAIDs should be avoided in ADHF.

When all else fails, UF for volume removal is a consideration for refractory congestion. The CARESS-HF trial examined the UF as an alternative to pharmacologic therapy in patients with $\mathrm{ADHF}$, persistent congestion, and worsening renal dysfunction. It showed the use of stepped-up pharmacologic therapy was superior to UF for the preservation of renal function at 96 hours and UF was associated with a higher rate of adverse events. It is usually reserved as a bailout therapy as there is limited evidence to support its use over loop diuretics. 


\section{Conclusions}

HF is major cause of morbidity and mortality with consistently increasing prevalence around the world. Even though there is paucity of data for mortality benefits, loop diuretics do provide significant clinical benefit in ADHF and help Improve symptoms. They remain primary diuretic of choice for treatment. IV form are more potent than oral formulation in ADHF. Thiazide diuretics are used in synergy with loop diuretics for sequential nephron blockade to overcome diuretic resistance. Aldosterone antagonist though carry a weak diuretic effect, have been shown to have mortality benefit in patients with ADHF. Understanding the cause of diuretic resistance remains pivotal to help reverse it. When all else fails, UF may be considered to help with volume removal.

\section{Acknowledgments}

Funding: None.

\section{Footnote}

Provenance and Peer Review: This article was commissioned by the Guest Editors (Dr. Debabrata Mukherjee and Dr. Jose B. Cruz Rodriguez) for the series "Heart Failure Update and Advances in 2021" published in Annals of Translational Medicine. The article was sent for external peer review organized by the Guest Editors and the editorial office.

Peer Review File: Available at http://dx.doi.org/10.21037/ atm-20-4600

Conflicts of Interest: Both authors have completed the ICMJE uniform disclosure form (available at http://dx.doi. org/10.21037/atm-20-4600). The series "Heart Failure Update and Advances in 2021" was commissioned by the editorial office without any funding or sponsorship. The authors have no other conflicts of interest to declare.

Ethical Statement: The authors are accountable for all aspects of the work in ensuring that questions related to the accuracy or integrity of any part of the work are appropriately investigated and resolved.

Open Access Statement: This is an Open Access article distributed in accordance with the Creative Commons
Attribution-NonCommercial-NoDerivs 4.0 International License (CC BY-NC-ND 4.0), which permits the noncommercial replication and distribution of the article with the strict proviso that no changes or edits are made and the original work is properly cited (including links to both the formal publication through the relevant DOI and the license). See: https://creativecommons.org/licenses/by-nc-nd/4.0/.

\section{References}

1. Schefold JC, Filippatos G, Hasenfuss G, et al. Heart failure and kidney dysfunction: epidemiology, mechanisms and management. Nat Rev Nephrol 2016;12:610-23.

2. Dalal R, Bruss ZS, Sehdev JS. Physiology, Renal Blood Flow and Filtration. Treasure Island (FL): StatPearls Publishing, 2020.

3. Felker GM, Ellison DH, Mullens W, et al. Diuretic Therapy for Patients With Heart Failure: JACC State-ofthe-Art Review. J Am Coll Cardiol 2020;75:1178-95.

4. Mueller C, Scholer A, Laule-Kilian K et al. Use of B-type natriuretic peptide in the evaluation and management of acute dyspnea. N Engl J Med 2004;350:647-54.

5. Januzzi JL Jr, Chen-Tournoux AA, Moe G. Aminoterminal pro-B-type natriuretic peptide testing for the diagnosis or exclusion of heart failure in patients with acute symptoms. Am J Cardiol 2008;101:29-38.

6. Dworzynski K, Roberts E, Ludman A, Mant J; Guideline Development Group of the National Institute for Health and Care Excellence. Diagnosing and managing acute heart failure in adults: summary of NICE guidance. BMJ 2014;349:g5695.

7. Binanay C, Califf RM, Hasselblad V, et al. Evaluation study of congestive heart failure and pulmonary artery catheterization effectiveness: the ESCAPE trial. JAMA 2005;294:1625-33.

8. Lala A, McNulty SE, Mentz RJ, et al. Relief and Recurrence of Congestion During and After Hospitalization for Acute Heart Failure: Insights From Diuretic Optimization Strategy Evaluation in Acute Decompensated Heart Failure (DOSE-AHF) and Cardiorenal Rescue Study in Acute Decompensated Heart Failure (CARESS-HF). Circ Heart Fail 2015;8:741-8.

9. Vaduganathan M, Greene SJ, Fonarow GC, et al. Hemoconcentration-guided diuresis in heart failure. Am J Med 2014;127:1154-9.

10. Zannad F, McMurray JJ, Krum H, et al. Eplerenone in patients with systolic heart failure and mild symptoms. N Engl J Med 2011;364:11-21. 
11. Roush GC, Kaur R, Ernst ME. Diuretics: a review and update. J Cardiovasc Pharmacol Ther 2014;19:5-13.

12. Mentz RJ, Stevens SR, DeVore AD et al. Decongestion strategies and renin-angiotensin-aldosterone system activation in acute heart failure. JACC Heart Fail 2015;3:97-107.

13. Ellison DH, Felker GM. Diuretic Treatment in Heart Failure. N Engl J Med 2017;377:1964-75.

14. Cosín J, Diez J, investigators T. Torasemide in chronic heart failure: results of the TORIC study. Eur J Heart Fail 2002;4:507-13.

15. Young M, Plosker GL. Torasemide: a pharmacoeconomic review of its use in chronic heart failure.

Pharmacoeconomics 2001;19:679-703.

16. Vasko MR, Cartwright DB, Knochel JP, et al. Furosemide absorption altered in decompensated congestive heart failure. Ann Intern Med 1985;102:314-8.

17. Cox ZL, Hung R, Lenihan DJ, et al. Diuretic Strategies for Loop Diuretic Resistance in Acute Heart Failure: The 3T Trial. JACC Heart Fail 2020;8:157-68.

18. Moranville MP, Choi S, Hogg J, et al. Comparison of metolazone versus chlorothiazide in acute decompensated heart failure with diuretic resistance. Cardiovasc Ther 2015;33:42-9.

19. Pitt B, Zannad F, Remme WJ, et al. The effect of spironolactone on morbidity and mortality in patients with severe heart failure. Randomized Aldactone Evaluation Study Investigators. N Engl J Med 1999;341:709-17.

20. Udelson JE, McGrew FA, Flores E, et al. Multicenter, randomized, double-blind, placebo-controlled study on

Cite this article as: Suri SS, Pamboukian SV. Optimal diuretic strategies in heart failure. Ann Transl Med 2021;9(6):517. doi: 10.21037/atm-20-4600 the effect of oral tolvaptan on left ventricular dilation and function in patients with heart failure and systolic dysfunction. J Am Coll Cardiol 2007;49:2151-9.

21. Felker GM, Mentz RJ, Cole RT, et al. Efficacy and Safety of Tolvaptan in Patients Hospitalized With Acute Heart Failure. J Am Coll Cardiol 2017;69:1399-406.

22. Wongboonsin J, Thongprayoon C, Bathini T, et al. Acetazolamide Therapy in Patients with Heart Failure: A Meta-Analysis. J Clin Med 2019;8:349.

23. Mullens W, Verbrugge FH, Nijst $P$, et al. Rationale and design of the ADVOR (Acetazolamide in Decompensated Heart Failure with Volume Overload) trial. Eur J Heart Fail 2018;20:1591-600.

24. Yancy CW, Jessup M, Bozkurt B, et al. 2013 ACCF/AHA guideline for the management of heart failure: a report of the American College of Cardiology Foundation/American Heart Association Task Force on Practice Guidelines. Circulation 2013;128:e240-327.

25. Felker GM, Lee KL, Bull DA, et al. Diuretic strategies in patients with acute decompensated heart failure. N Engl J Med 2011;364:797-805.

26. Salvador DR, Rey NR, Ramos GC, et al. Continuous infusion versus bolus injection of loop diuretics in congestive heart failure. Cochrane Database Syst Rev 2005;(3):CD003178.

27. Kitsios GD, Mascari P, Ettunsi R, et al. Co-administration of furosemide with albumin for overcoming diuretic resistance in patients with hypoalbuminemia: a metaanalysis. J Crit Care 2014;29:253-9. 